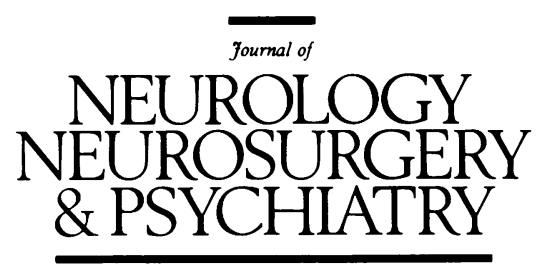

Editorial

\title{
The idiopathic inflammatory myopathies and their treatment
}

The inflammatory myopathies are the largest group of acquired myopathies of adult life and may also occur in infancy and childhood. They have in common the presence of inflammatory infiltrates within skeletal muscle, usually in association with muscle fibre destruction. They can be subdivided into those which are due to known viral, bacterial, protozoal or other microbial agents and those in which no such agent can be identified and in which immunological mechanisms have been implicated. ${ }^{1}$ The latter group includes polymyositis, dermatomyositis and inclusion body myositis. The evidence for an autoimmune aetiology consists of: 1) an association with other autoimmune diseases; 2) serological tests which reflect an altered immune state; and 3) the responsiveness of polymyositis and dermatomyositis, if not of the inclusion body variety, to immunotherapy. ${ }^{2}$ Polymyositis may rarely occur in infancy (infantile myositis) but is commoner in adult life, while dermatomyositis can occur both in children and in adults and is often associated with other connective tissue diseases, with immune deficiency states or with other systemic disorders; in middle or late life there is a statistical association with malignant disease. Both polymyositis and dermatomyositis can sometimes be druginduced. $^{3}$

Related disorders include sarcoid myopathy and granulomatous myositis (which can only be distinguished from sarcoid myopathy by the absence of evidence of sarcoid in tissues and organs other than muscle), localised nodular myositis, ${ }^{4}$ eosinophilic polymyositis (which occurs as part of a systemic hypereosinophilic syndrome characterised by eosinophilia, anaemia, hypergammaglobulinaemia, cardiac and pulmonary involvement, skin changes, peripheral neuropathy and encephalopathy ${ }^{5}$ ) and eosinophilic perimyositis. ${ }^{67}$ Focal and relapsing forms of eosinophilic myositis have been described, ${ }^{8}$ as has a form of angiopathic myositis, sometimes associated with polyarteritis nodosa, Churg-Strauss vasculitis or Wegener's granulomatosis. ${ }^{1}$ Eosinophilic fasciitis may also be confused with the myositic syndrome; in this condition there is painful swelling and induration of the skin and soft tissues of the extremities and trunk leading to weakness and contractures in the limbs, often with a marked eosinophilia in the peripheral blood and hypergammaglobulinaemia. ${ }^{9} \mathrm{~A}$ form of eosinophilic fasciitis presenting as a sclerodermalike syndrome, now called the tryptophan-eosinophiliamyalgia syndrome, has also been reported in recent years in patients taking L-tryptophan for the treatment of depression or insomnia ${ }^{10}$ but is thought by some to be due to a contaminant in the L-tryptophan rather than to a direct effect of the drug itself. A resemblance has been suggested between this condition and the so-called Spanish toxic oil syndrome. ${ }^{11}$ In some such cases a peripheral neuropathy also occurs. ${ }^{12}$
As new knowledge has accumulated over the course of the last 10 years, it has become increasingly clear that there are distinct pathological and immunological differences between polymyositis on the one hand and dermatomyositis on the other, though in some cases there is clearly an overlap between the two conditions. In polymyositis there is usually scattered necrosis of single muscle fibres which appear hyalinised in the early stages and are subsequently invaded by mononuclear phagocytic cells. Regenerating fibres are usually seen singly or in small groups distributed focally and randomly throughout the muscle. The inflammatory cell infiltrate is predominantly intrafascicular (endomysial) surrounding muscle fibres rather than in the interfascicular septa, though perivascular infiltrates may also be found; the cellular infiltrate consists mainly of lymphocytes, plasma cells and macrophages. Immunocytochemical studies using monoclonal antibody markers have shown that most of the lymphocytes and the cellular infiltrates are activated $T$ cells with a mixture of $\mathrm{CD} 8+$ (suppressor/cytotoxic) and $\mathrm{CD} 4+$ (helper/ inducer) cells, as well as macrophages..$^{1314}$ Class 1 MHC antigen expressed on the surface of non-necrotic muscle fibres which are being invaded by lymphocytes supports the view that the muscle fibre damage is caused by cytotoxic $\mathrm{T}$ lymphocytes as these cells require the presence of Class $1 \mathrm{MHC}$ molecules on target cells to exert their action. ${ }^{2}$

In dermatomyositis, particularly in childhood, isolated muscle fibre necrosis is a rare finding; more often, portions of a muscle fasciculus may be necrotic and the lesions resemble micro-infarcts. There is also a typical perifascicular atrophy involving both type 1 and type 2 muscle fibres. Microvascular changes, including capillary loss, necrosis and thrombosis of capillaries, arterioles and venules accounting for the appearances resembling microinfarction are also common. ${ }^{1516}$ Immunoglobulins and complement components, including the C5b-9 membrane attack complex, are commonly found in blood vessel walls, suggesting that the condition is an immune complex vasculopathy. ${ }^{16-18}$ Cellular infiltrates in this condition are principally perimysial and perivascular and analysis of mononuclear cell subsets has shown a high percentage of $B$ cells and a high CD4 $+/ \mathrm{CD} 8+\mathrm{T}$ cell ratio in perivascular and perimysial sites. It is suggested that in this condition there is a helper $\mathrm{T}$ cell-dependent stimulation of B cells to secrete immunoglobulins. ${ }^{19}$

Inclusion body myositis, by contrast, is a distinct variety of inflammatory myopathy, not generally associated with skin change, malignancy or collagen vascular disease, though there have been occasional reports of an association with Sjögren's syndrome, scleroderma, immune thrombocytopenia, coeliac disease, sarcoidosis and malignancy. ${ }^{1}$ The weakness is slowly progressive, usually pain- 
ful, generally beginning in distal as well as proximal limb muscles, occurring principally in males; usually the condition is slowly progressive over a period of $10-20$ years. Muscle biopsy reveals bluish granular inclusions distributed round the edge of slit-like vacuoles (rimmed vacuoles) in muscle fibres; inflammatory cell infiltrates are predominantly endomysial and less frequently perimysial and perivascular in location. Electron microscopy in this condition invariably reveals intranuclear and cytoplasmic masses of filamentous microtubules. ${ }^{20}$ While attempts at viral isolation have been uniformly unsuccessful, Chou ${ }^{21}$ reported immunostaining for mumps virus antigens in the nuclear and cytoplasmic inclusions in eight cases and postulated that the condition is due to a chronic persistent mumps virus infection. However, Nishino and others ${ }^{22}$ have failed to confirm that finding. Hohlfeld and Engel ${ }^{23}$ have recently reported results consistent with the hypothesis that the cytotoxic $T$ cell response in this condition is directed against viral antigens, but the question of whether it results from one or more specific viruses is still uncertain. For the pathological diagnosis of this condition, at least one rimmed vacuole and at least one group of atrophic fibres must be seen per high-power field, and there must be an endomysial inflammatory infiltrate withlymphocytes invading non-necrotic muscle fibres as well as an electronmicroscopic demonstration of the typical filamentous inclusions. ${ }^{20}$

Turning now to treatment, the response to steroid drugs and immunotherapy in inclusion body myositis has been almost invariably disappointing. While a few patients appear to show temporary improvement, and in some it has been suggested that the disease process may have been slowed down or arrested, most authors have concluded that the condition does not respond to such treatment and trials of antiviral agents have also been disappointing. ${ }^{24}$ In polymyositis and dermatomyositis, by contrast, the results of treatment with steroids and immunosuppressive agents have been so strikingly successful that since the 1950 s it has never been possible to carry out a double-blind controlled trial of treatment in either condition. Such trials that have been performed have therefore had to depend upon withinpatient comparisons or sequential analysis of the results of treatment, or more particularly upon the comparison of the outcome in treated patients on the one hand with that observed in patients previously seen who were untreated before the use of steroids became widespread. The latter type of trial, based upon retrospective analysis has, however, proved unsatisfactory in most instances because the criteria for diagnosis of polymyositis and dermatomyositis were relatively imprecise in previous years, before steroids were generally used. ${ }^{25}$ Nevertheless, from the 1970 s onwards in both conditions most authorities have advocated the addition of immunosuppressive agents to high-dose steroid therapy; azathioprine has been generally favoured, though some workers have preferred methotrexate or cyclophosphamide. In 1981 Bunch $^{26}$ carried out a double-blind trial in adults with dermatomyositis, comparing prednisone plus azathioprine with prednisone alone, and although there was no short-term benefit, he was satisfied that the long-term disability was diminished in those given both remedies. In severe childhood dermatomyositis with vasculitis he recommended $2 \mathrm{mg}$ per $\mathrm{kg}$ of prednisone parenterally four times daily, continuing the treatment in high dosage for a month and then giving alternate-day oral treatment. He found that chelating agents were ineffective in reducing calcinosis in childhood polymyositis but probenecid appeared to reduce calcium deposits in some cases.

Among others, Sarnat, ${ }^{27}$ and Rosenberg and Ringel ${ }^{28}$ have confirmed the beneficial effect of immunosuppressive agents such as azathioprine, methotrexate and cyclophosphamide when given in addition to steroids. Cyclosporin A has proved successful in a few resistant cases, but Grezard and others ${ }^{29}$ reported that a severe myopathy resulted from cyclosporin treatment in a patient who had previously undergone a renal transplant. Recently Engel and Emslie-Smith ${ }^{30}$ have found intravenous pulsed methyl-prednisolone to be superior in its effects to oral treatment in both polymyositis and dermatomyositis but have suggested that such treatment should only be continued for a few weeks and should be followed by combined oral steroids given on an alternate-day basis with an appropriate immunosuppressive remedy for longer-term maintenance treatment. Yet again, in some resistant cases total body irradiation or total lymphoid irradiation has been recommended and has been found successful by Engel $^{31}$ and others. More recently Cherin and others, ${ }^{32}$ writing from Paris, reported strikingly beneficial effects in both polymyositis and dermatomyositis using intravenous immunoglobulin. Ten patients received $1 \mathrm{~g}$ per $\mathrm{kg}$ daily for two days each month while five received $0.5 \mathrm{~g}$ per $\mathrm{kg}$ daily for five days each month, the mean course of treatment being four months. This treatment was given with prednisone in 11 cases, methotrexate in five, and plasmapheresis in one. Marked improvement was noted in 13 of the 15 cases so treated; the authors concluded that intravenous immunoglobulin might well be used to replace or reduce steroid and immunosuppressive drug treatment in such cases. Finally, it should be mentioned that in dermatomyositis, following the report of Bennington and Dau in $1981,{ }^{33}$ plasmapheresis has been shown to be effective, but most authorities still reserve this form of treatment for use in severe cases unresponsive to oral steroids and immunosuppressive agents.

The present position therefore appears to be that in both polymyositis and dermatomyositis the treatment of choice is still oral prednisone combined with azathioprine. Once the disease has been brought under control, as demonstrated by clinical and functional assessment and a fall in serum creatine kinase activity, it is reasonable to move to alternate-day treatment with prednisone, while continuing with the azathioprine for 12-18 months, thereafter using prednisone in whatever level of dosage is required to control the clinical manifestations of the disease for as long as may be needed (this may be many years). It is possible that pulsed methyl-prednisolone as an initial treatment may prove superior to oral treatment, but this is still uncertain. The place of intravenous immunoglobulin is still to be evaluated but this is unlikely to be widely used except in resistant cases for as long as it proves to be so horrendously expensive (at present about $£ 2000$ for a course). Plasmapheresis in dermatomyositis and wholebody or lymphoid irradiation in polymyositis should be kept in reserve for use only in cases resisting standard treatment.

JOHN WALTON (LORD WALTON OF DETCHANT)

1 Mastaglia FL, Walton JN. Inflammatory myopathies. In: Mastaglia FL Walton JN, eds. Skeletal muscle pathology, 2nd ed. Edinburgh: Churchill Livingstone, 1991:360-92.

2 Engel AG. Immune effector mechanisms in the idiopathic inflammatory myopathies. J Neurol Sci 1990;98(suppl):6.

3 Mastaglia FL, ed. Inflammatory diseases of muscle. Oxford: Blackwell, 1988 : $110-11$.

4 Cumming WJK, Weiser R, Teoh R, Hudgson P, Walton JN. Localised nodular myositis: a clinical and pathological variant of polymyositis. Quart J Med 1977;46:531-46.

5 Layzer RB, Shearn MA, Satya-Murti S. Eosinophilic polymyosicis. Ann Neurol 1977;1:65-71.

6 Serratrice G, Pellissier JF, Cros D, Gastaut JL, Brindisi G. Relapsing eosinophilic perimyositis. J Rheumatol 1980;7:199-205.

7 Lakhanpal S, Duffy J, Engel AG. Eosinophilia associated with perimyositis and pneumonitis. Mayo Clinic Proc 1988;63:37-41.

8 Kamm MA, Dennett X, Byrne E. Relapsing eosinophilic myositis-a cause 
of pseudothrombophlebitis in an alcoholic. $J$ Rheumatol 1987;14:831-4. Shulman LE. Diffuse fasciitis with hypergammaglobulinaemia and eosinophilia: a new syndrome? J Rheumatol 1974;1:46-54.

10 Glasberg MR. Eosinophilic myositis: an expression of L-tryptophan toxicity. J Neurol Sci 1990;98(suppl):177.

11 Deighton CM. Eosinophilia myalgia syndrome. BMJ 1990;301:611.

12 Smith BE, Dyck PJ. Peripheral neuropathy in the eosinophilia-myalgia syndrome associated with L-tryptophan ingestion. Neurology 1990;40: 1035-40.

13 Arahata K, Engel AG. Monoclonal antibody analysis of mononuclear cells in myopathies. I. Quantitation of subsets according to diagnosis and sites of accumulation and demonstrations and counts of muscle fibers invaded by accumulation and demonstrations and

14 Arahata K, Engel AG. Monoclonal antibody analysis of mononuclear cells in myopathies. III. Immunoelectron microscopy aspects of cell-mediated muscle fiber injury. Ann Neurol 1986;19:112-5.

15 Carpenter S, Karpati G. The major inflammatory myopathies of unknown cause. Pathology Annual 1981;16:205.

16 Emslie-Smith AM, Engel AG. Microvascular changes in early and advanced dermatomyositis: a quantitative study. Ann Neurol 1990;27:343-56.

17 Whitaker JN, Engel WK. Vascular deposits of immunoglobulin and complement in idiopathic inflammatory myopathy. New Engl J Med 1972;286:333-8.

18 De Visser M, Emslie-Smith AM, Engel AG. Early ultrastructural alterations in adult dermatomyositis. Capillary abnormalities precede other structural changes in muscle. J Neurol Sci 1989;94:181-92.

19 Engel AG, Arahata K. Mononuclear cells in myopathies: quantitation of functionally distinct subsets, recognition of antigen-specific cell-mediated functionally distinct subsets, recognition of antigen-specific cell-mediated cytotoxicity in some diseases, and implications for the pathogenesis of the
different inflammatory myopathies. Human Pathology 1986;17:704-21.
20 Lotz BP, Engel AG, Nishino H, Stevens JC, Litchy WJ. Inclusion body myositis. Brain 1989;112:727-47.

21 Chou SM. Inclusion body myositis: a chronic persistent mumps myositis? Human Pathology 1986;17:765-77.

22 Nishino H, Engel AG, Rima BK. Inclusion body myositis: the mumps virus hypothesis. Ann Neurol 1989;25:260-4.

23 Hohlfeld R, Engel AG. Inclusion body myositis: functional and phenotypic properties of $\mathrm{T}$ cells isolated from muscle. J Neurol Sci 1990 98(suppl): 109.

24 Chou SM. Viral myositis. In: Mastaglia FL, ed. Inflammatory diseases of muscle. Oxford: Blackwell, 1988:125-53.

25 Walton JN. Therapeutic trials: myositis. J Neurol Sci 1990;98(suppl):3.

26 Bunch TW. Prednisone and azathioprine for polymyositis: long-term follow-up. Arthritis Rheum 1981;24:45-8.

27 Sarnat HB. Juvenile dermatomyositis. In: Mastaglia FL, ed. Inflammatory diseases of muscle. Oxford: Blackwell, 1988:71-86.

28 Rosenberg NL, Ringel SP. Adult polymyositis and dermatomyositis. In: Mastaglia FL, ed. Inflammatory diseases of muscle. Oxford: Blackwell, 1988:87-106.

29 Grezard O, Lebranchu Y, Birmele B, Sharobeem R, Nivet H, Bagros P. Cyclosporin-induced muscular toxicity. Lancet 1990;335:177.

30 Engel AG, Emslie-Smith AM. Inflammatory myopathies. Current Opinion Neurol Neurosurg 1989;2:695-9.

31 Engel WK, Lichter AS, Galdi AP. Polymyositis: remarkable response to total body irradiation. Lancet $1981 ; \mathbf{i}: 658$.

32 Cherin $\mathrm{P}$, Herson S, Wechsler B, et al. Intravenous immunoglobulin for polymyositis and dermatomyositis. Lancet 1990;335:116.

33 Bennington JL, Dau PC. Patients with polymyositis and dermatomyositis who undergo plasmapheresis therapy. Pathologic findings. Arch Neurol 1981;38:553-60.

\section{Neurological stamp}

\section{CLAUDIUS GALEN 131-201 AD}

After studying at the best medical schools of the time, Galen returned initially to his native city, Pergamon and was physician to the gladiators. There he would have had ample opportunity to observe the effects of acute injuries to the head and spine. Galen, who did not leave any good accounts of clinical cases, only miraculous cures, had an answer for every problem. His dogmatism and infallibility persisted for 14 centuries until Vesalius (1514-64). Observations on the anatomy of animals were transferred to the human anatomy but he lamented the prejudice which prevented dissection of the human body. Neurology was the best feature of his anatomical work. Most of the gross structures of the brain were classified by him. He knew of seven pairs of cranial nerves and of the cervical, brachial and lumbar sacral plexuses. The sympathetic ganglia were described as reinforcers of the nerves. His myology was based mainly on the study of the barbary ape. He understood the difference between origin and insertion, and was aware of most muscles and their functions:

Among his brilliant experiments were the demonstrations of the function of the laryngeal nerves, the motor and sensory functions of the spinal nerve roots, and the effect of transverse incision and hemisection of the spinal cord. He knew hydrophobia followed the bite of a mad dog and sometimes maniacal attacks supervened. Galen showed that arteries contained blood and not air and was close to discovering the circulation of the blood. $\mathrm{He}$ was the founder of experimental physiology. This Hungarian stamp issued in 1989 is one of a "Pioneers of Medicine" series.

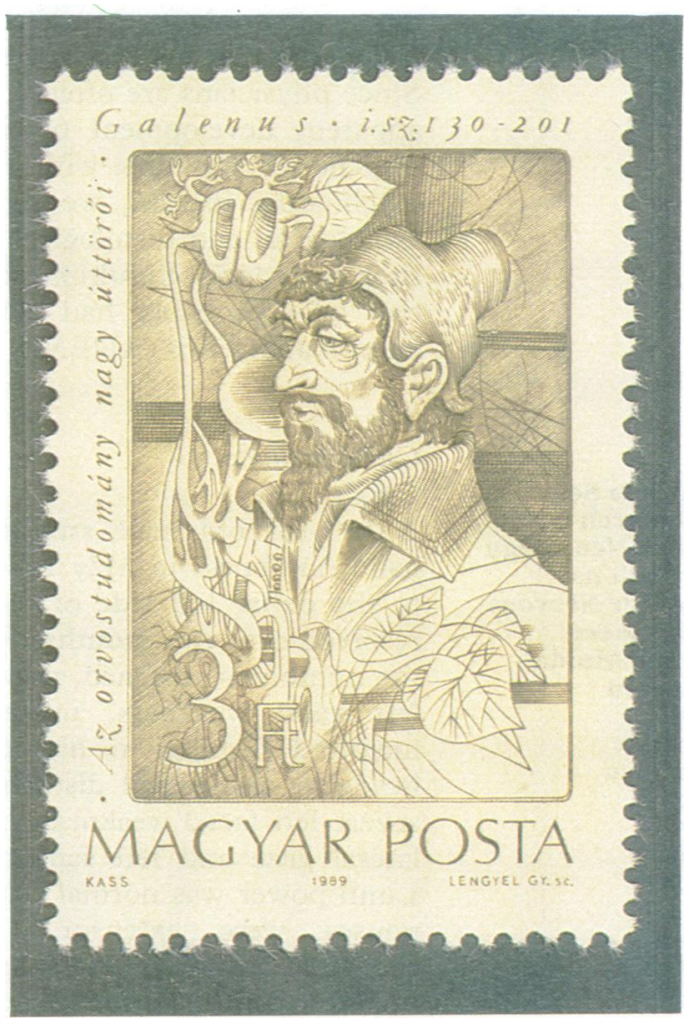

